\title{
Les baguettes à moissonner en Caucase
}

The harvest reaping-stick in Georgia (Caucasus)

Las « varillas de cosechar » del Cáucaso

\section{Pascal Reigniez}

\section{(2) OpenEdition}

\section{Journals}

Édition électronique

URL : https://journals.openedition.org/tc/69

DOI : $10.4000 /$ tc. 69

ISSN : 1952-420X

Éditeur

Éditions de l'EHESS

Édition imprimée

Date de publication : 1 décembre 2003

Pagination : 91-114

ISSN : 0248-6016

Référence électronique

Pascal Reigniez, «Les baguettes à moissonner en Caucase », Techniques \& Culture [En ligne], 41 | 2003 , mis en ligne le 13 janvier 2005, consulté le 29 septembre 2022. URL : http://journals.openedition.org/ tc/69; DOI : https://doi.org/10.4000/tc.69

Ce document a été généré automatiquement le 29 septembre 2022.

Tous droits réservés 


\title{
Les baguettes à moissonner en Caucase
}

\author{
The harvest reaping-stick in Georgia (Caucasus) \\ Las « varillas de cosechar » del Cáucaso
}

Pascal Reigniez

1 La Géorgie est une République indépendante ${ }^{2}$ d'environ cinq millions et demi d'habitants qui s'étend au sud de la chaîne des hautes montagnes du Caucase sur une superficie de $69700 \mathrm{~km}^{2}$. Le climat y est subtropical, la géographie physique est schématiquement constituée de deux forts massifs, le Grand Caucase au nord, et le Petit Caucase au sud, dominant des terres vallonnées d'altitude variable. Parmi les outils agricoles employés dans ce pays jusqu'au siècle dernier, il en est un, formé de deux baguettes de bois, qui servait à récolter certaines espèces de blé. Cet instrument, dénommé localement chnakvi, mal connu et tout à fait intéressant, pose aux ethnologues quelques problèmes qu'il serait prématuré de considérer comme résolus (Reigniez s/presse).

\section{La problématique et les méthodes}

2 La disparition rapide du chnakvi, observée au cours des cinquante dernières années, est probablement due à une conjonction de facteurs dont on peut supposer qu'ils sont principalement d'ordre économique. Le plus marquant d'entre eux fut peut-être l'emprise de l'ex-URSS sur la Géorgie et notamment, deux éléments aux effets d'autant plus rapides qu'ils se sont cumulés: en premier lieu, l'obligation faite aux communautés villageoises de se conformer au système de production soviétique en abandonnant les formes d'exploitation traditionnelles et pluriséculaires qui les caractérisaient; en second lieu, il s'est produit, par nécessité économique, une importation massive de blé russe sur les marchés caucasiens, d'où une relative inutilité de certaines cultures, pas assez productives -en particulier les espèces difficiles à 
travailler comme les blés zanduri et makha- qui furent abandonnées avec les techniques de récolte attenantes.

3 Le principe de fonctionnement du chnakvi repose sur l'emprisonnement puis l'arrachement vertical des épis de blé ou d'orge de leurs tiges, les plantes étant encore sur pied dans le champ. Il convient sur ce point de souligner que cet outil fonctionnait en arrachant, et non pas en érussant ${ }^{3}$. Ces deux actions sont également différentes du geste qui consiste simplement à cueillir les épis à la main, en les cassant. Les baguettes permettaient d'enlever simultanément plusieurs épis. Pareille technique, identifiée à l'arrachage parce que très proche de lui n'a, en fait, pas de signification propre (François Sigaut, com. pers.), et nous souhaitons la définir par le néologisme de désépiage, en rapport avec le verbe désépier ${ }^{4}$, qui décrit l'extraction stricto sensu d'un épi, tout en offrant la possibilité de préciser si l'action est effectuée à la main ou avec un outil, sur un seul épi ou sur plusieurs.

4 En Géorgie, le rapport entre la plante et le chnakvi est direct, ce qui justifie une cartographie plausible; mais l'effet négatif induit par un rapprochement aussi simple pourrait faire justement ressortir que ce rapport était exclusif, alors que précisément, nous savons que ce ne fut pas le cas. En effet, certains Géorgiens âgés nous ont décrit un instrument assimilé au chnakvi, qui fut employé dans le sud-ouest du pays (région d'Adjari, près de Batoumi, zone de la Mer Noire) pour récolter une espèce de blé que l'on appelle khaci pouri en géorgien. Cette céréale présente des caractéristiques très proches des espèces de blé zanduri et makha.

5 L'académicien géorgien Georges Chitaïa $(1987: 131)$ parle d'ailleurs de cet outil comme étant très similaire au chnakvi, à savoir constitué de deux baguettes reliées par l'une de leurs extrémités, mais d'une longueur inférieure (entre 25 et 35 centimètres), ce qui semble assez court. L'auteur précise que l'objet, nommé artochani, pouvait être fabriqué en bois de châtaignier, de hêtre ou d'épicéa.

6 Nous retiendrons que l'approche anthropologique confirme l'existence de deux outils servant à moissonner par désépiage, aux noms différents, en usage dans plusieurs régions du même pays, sans qu'il soit pour l'instant possible de préciser un rapport d'antériorité ni l'origine de la relation que chaque forme entretient avec la plante travaillée. Toutefois, signaler cette concomitance constitue déjà un fait important, qui n'a été observé nulle part ailleurs - là où est connue cette technique de récolte des céréales.

7 Il faut également noter que le principe de fonctionnement du chnakvi se retrouve dans une autre technique géorgienne, celle de la récolte du miel, pour laquelle on employait autrefois et selon les endroits un outil spécifique, ressemblant au chnakvi, dont les baguettes, longues de 35 à $40 \mathrm{~cm}$, étaient rattachées à l'une de leurs extrémités par un lien souple qui maintenait un petit écartement. Présent dans les régions de Guria et de Samegrelo (Chitaïa 1987 : 131), il portait en Mingréli le nom de chanko ou chanki. Pour extraire le produit, l'apiculteur découpait le rayon (miel et cire) puis mettait les morceaux ainsi obtenus dans un sac de toile qu'il refermait et suspendait à une faible hauteur. De cette manière, le sac restait relativement libre, suspendu au-dessus d'un récipient à large bord. Ensuite, l'opérateur écartait les deux baguettes au niveau de la partie haute du sac, sur laquelle il les réunissait. A ce stade de l'opération, il maintenait le chanko par chacune de ses extrémités, puis exerçait des pressions successives plus ou moins appuyées, avec un déplacement simultané de l'outil du haut vers le bas du sac, le 
geste étant répété et modulé afin d'exprimer le miel, c'est-à-dire de le faire passer au travers des mailles du tissu ${ }^{5}$.

8 Un autre outil, également dénommé chanko, constitué cette fois de trois baguettes grossièrement taillées réunies naturellement ou artificiellement par l'une de leurs extrémités au moyen d'un lien serré, mais qui autorisait une certaine souplesse, servait à la récolte de la cire : le produit était placé dans un sac et fréquemment arrosé d'eau très chaude, et l'on pressait le tout pour l'en faire sortir. Les pressions sur les trois branches libres étaient exercées simultanément, comme si l'on cherchait à les réunir pour s'en servir comme une sorte de pressoir. Il s'agit là de l'un des seuls outils de ce genre, à trois branches distinctes, dont nous ayons connaissance (Reigniez s/presse ; voir tableau en annexe).

ous ne pouvons que constater l'existence d'une certaine similitude entre les techniques que nous venons de décrire ${ }^{6}$. Si pour les moissons le chnakvi et l'artochani fonctionnaient sur le principe de l'arrachage vertical (de bas en haut), le chanko double (ou chanki) fonctionnait de haut en bas pour presser et faire descendre la substance ductile. C'est le principe même des baguettes qu'il est important de relever ici, dans la mesure où il apparait caractéristique de deux produits récoltés en Géorgie, l'un solide, l'autre relativement liquide.

10 Aussi, puisque des outils très semblables ont été employés pour assurer deux opérations distinctes, il s'avère désormais difficile de ne pas faire de rapprochements, même s'il faut en nuancer les termes, car chaque technique est le résultat d'une adaptation. On pourrait peut-être établir une localisation géographique et chronologique plus précise encore et, par suite, définir l'antériorité d'un outil sur l'autre, comme l'incidence possible de l'un sur l'autre. La question d'une incidence plus ou moins notable d'une technique sur l'autre doit se poser mais il est prématuré d'y répondre, en l'état actuel des recherches.

11 Ajoutons que cette technique de moisson du blé a été relevée par d'autres scientifiques dans deux autres endroits du monde : le nord de l'Espagne, et certaines hautes vallées du Népal. La voie comparative que nous empruntons, en témoignant par ailleurs de la rareté du phénomène, articule notre problématique: aussi notre article vient-il s'ajouter comme un troisième élément aux travaux déjà parus ${ }^{7}$.

Dans le nord de l'Espagne, c'est un type d'outil observé en Asturies au cours de la première moitié $\mathrm{du} \mathrm{XX}^{\mathrm{e}}$ siècle par le savant russe Nicolaï Vavilov ${ }^{8}$ lors d'un voyage scientifique qu'il entreprit en Europe, et qui attira l'attention des ethnologues européens. Bien plus tard, en septembre 1979, un groupe de scientifiques, à l'initiative de François Sigaut, se rendit en Asturies où l'observation complète et précise du fonctionnement des baguettes pour la récolte des blés vêtus (épautre) dans les villages de Zureda et de Santibañez de la Fuente, a pu être consignée et filmée, mettant l'objet en valeur (Sigaut 1978).

13 Au sein d'une réflexion d'ensemble, François Sigaut suppose, d'une part que cet outil était probablement plus répandu autrefois dans plusieurs régions du monde, et d'autre part, qu'il existe un lien entre l'outil et le végétal récolté, ce qui reste en accord avec les hypothèses émises par Vavilov (Sigaut et al. 1980). Les baguettes observées dans les Asturies mesurent environ $60 \mathrm{~cm}$, leur section est importante (entre 2 et $2,5 \mathrm{~cm}$ ), irrégulière, ovoïde, ou parfois circulaire. Elles possèdent le plus souvent une zone proximale de préhension qui peut être gravée, alors que la zone distale ne l'est pas; 
elles sont donc assez approchantes des baguettes du Caucase, mais typologiquement bien distinctes.

Un autre type de baguettes, cette fois morphologiquement et typologiquement beaucoup plus proches par leur conception et par leur maniement de celles utilisées en Géorgie, a été signalé au cours des dernières décennies pour les populations de quelques hautes vallées du Népal central, dans les régions de Langthang, du Mustang ainsi que pour le groupe Tamang vivant dans la haute Ankhu Khola, qui récoltait encore, il y a une vingtaine d'années, l'orge et le blé de cette manière (Toffin 1983).

Les zones de répartition probables du chnakvi en Géorgie depuis le XIX ${ }^{\mathrm{e}}$ siècle semblent aujourd'hui pouvoir être situées; la cartographie correspond globalement -et c'est finalement logique- à celle des blés zanduri et makha. On le retrouve essentiellement dans l'une des anciennes régions de la partie occidentale du pays (Mingréli) et dans le nord-ouest (voir carte). La cartographie précise de cet instrument reste toutefois assez ardue à dresser d'après les rares témoignages ethnographiques et le peu de documents dont on dispose. Au cours du XX siècle cependant, de bonnes localisations ont été effectuées par des ethnologues géorgiens grâce à des éléments concrets. Ainsi, G. Chitaïa (1980: 75) repère l'outil dans trois régions du pays: Adjara, Lechkhumi et Mingréli. Mais les aires d'utilisation sont plus difficiles à déterminer en ce qui concerne les périodes antérieures au XIX ${ }^{\mathrm{e}}$ siècle.

\section{Les analyses et les hypothèses}

Dans l'ouest du Caucase, c'est le terme de chnakvi qui désigne le plus couramment l'outil. Il existe néanmoins plusieurs variantes phonétiques de ce terme. Les principales sont samkvi, sankvi, shakvi ou chamkvi. Ce mot serait, selon une hypothèse soutenue par certains spécialistes et parce qu'il existerait des rapports d'ordre étymologique, à rapprocher d'un mot beaucoup plus ancien, chinakoû, issu de la langue Svane et qui signifierait "brasser, prendre à bras le corps", sans que soit pour autant précisée l'existence d'un type particulier d'outil en connexion avec ce dernier terme (Bregadze 1982 : 86). Il est intéressant de confronter ce point de vue à une seconde hypothèse qui réunit des spécialistes géorgiens proposant de rapprocher ces termes des matériaux linguistiques de l'Orient ancien, plus précisément de la langue akkadienne ${ }^{9}$, où le mot sanki signifiait « presser, couper ».

En l'état actuel de la question, il nous semble prématuré de trancher, même si la seconde hypothèse nous paraît plus séduisante que la première; elle correspond en effet plus directement à l'action obtenue avec le chanko (ou chanki) double et triple, alors que l'on fait plus difficilement le lien avec l'action de saisir des gerbes, surtout lorsque ce sont les épis seuls qui ont été récoltés. Mais la solution, qui doit tenir compte de paramètres plus vastes, n'est pas si évidente ${ }^{10}$. 


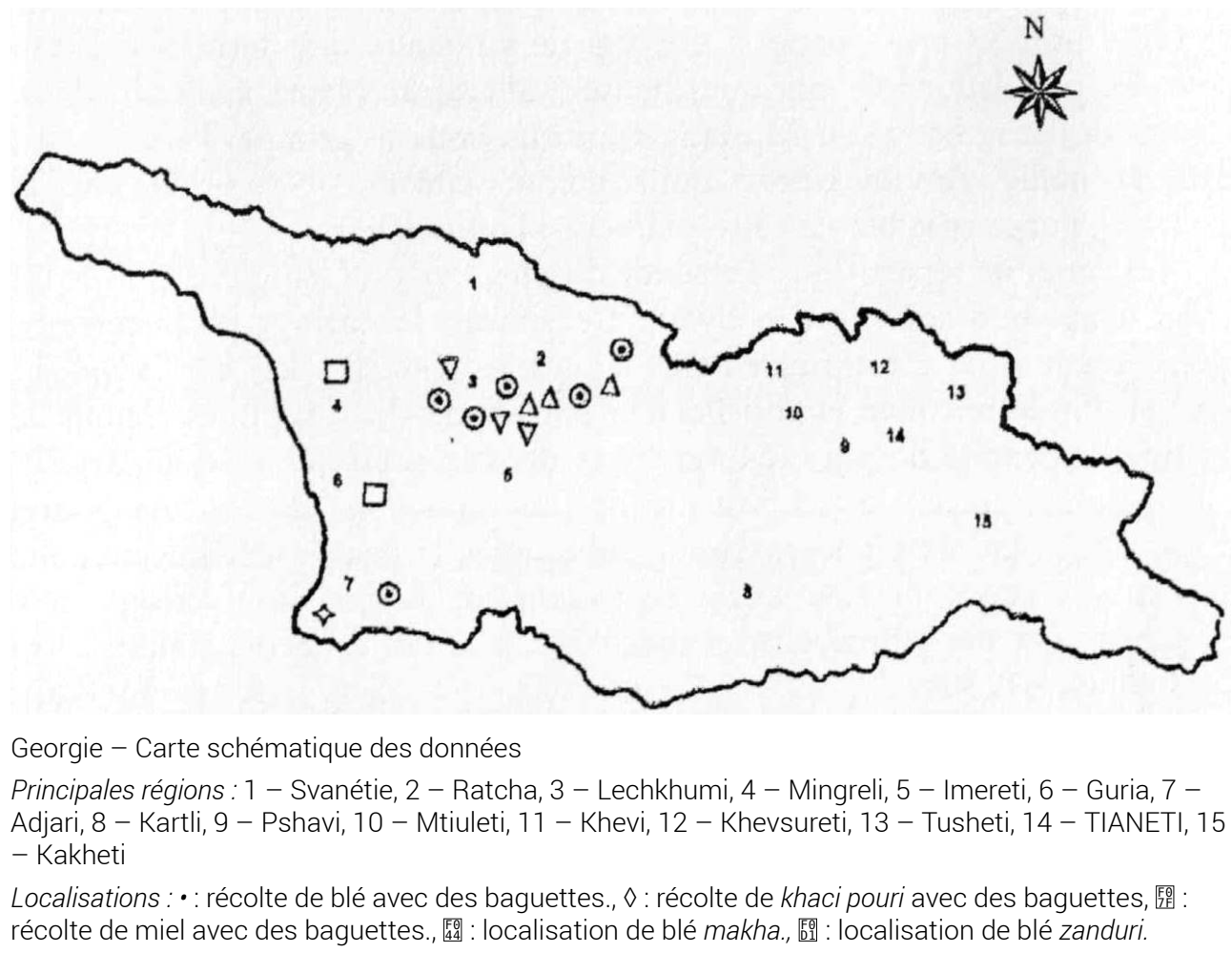

\section{La fabrication du chnakvi}

Le chnakvi se compose de deux baguettes de bois, d'une section circulaire d'environ de 1 à $1,5 \mathrm{~cm}$ et d'une longueur à peu près égale (environ $50 \mathrm{~cm}$ ). Il importe peu que les deux baguettes soient très exactement de la même longueur ou strictement rectilignes. Elles sont attachées sur l'une de leurs extrémités par un lien souple qui peut être fixé de deux manières : soit en traversant diamétralement le bois préalablement percé, soit -et c'est la technique la plus commune- en nouant le lien autour du bois, celui-ci étant alors marqué d'une légère incision afin de mieux le recevoir. Autrefois, ce lien était en peau (cuir) ou bien en fibres végétales tressées, mais depuis le XIX ${ }^{\mathrm{e}}$ siècle, les paysans ont employé de la cordelette solide (voire d'étroites bandelettes de tissu). D'une longueur de 8 à $10 \mathrm{~cm}$ lorsqu'il était tendu, il déterminait ainsi l'écartement maximal de l'une des extrémités des baguettes. On notera que certains auteurs mentionnent des chnakvi dont l'écartement maximal variait de 3 à $3,5 \mathrm{~cm}$ (Dekaprelevich \& Menabde 1932:5, 1, 44), ce que nous avons pu observer sur l'un de ces outils, conservé au Département d'Ethnographie du Musée Djanachia de l'État de Géorgie à Tbilissi. Il est possible que ce lien souple ait eu pour fonction d'assurer une meilleure préhension du chnakvi, dans la mesure où il se trouvait placé du côté de la main directrice. Il assurait probablement une manipulation plus aisée permettant d'obtenir l'écartement voulu à l'autre extrémité, dépourvue en revanche de toute attache. On peut également supposer - et cela n'exclut pas la première remarque-que ce lien servait à solidariser les baguettes, afin d'éviter que l'une d'elles ne tombe lors de la manipulation de l'outil, ce qui pouvait faire perdre du temps à l'utilisateur, nous avons en effet remarqué que les baguettes fines de Géorgie (en bois), du Népal (en bois et bambou), de faible section sont souvent reliées alors que les baguettes plus grandes 
et de section plus importantes ne le sont généralement pas (outil des Asturies), mais cela ne semble cependant pas systématique.

Deux des chnakvi conservés au Musée de l'État de Géorgie à Tbilissi, que nous ont présentés les scientifiques géorgiens ${ }^{11}$, fournissent certaines informations. Ainsi, le premier de ces deux outils, le plus court, est fabriqué avec deux baguettes, l'une mesurant $47 \mathrm{~cm}$ de long, la seconde $48 \mathrm{~cm}$; elles sont reliées par une corde assez serrée, au point que l'écartement possible entre les deux bois est assez réduit (fig.1). Les extrémités libres des deux baguettes sont taillées en pointe longue (fig. 2), alors que les extrémités attachées sont taillées en têtes très courtes, à la lame et vraisemblablement au couteau (fig. 3). Un élément intéressant est que l'une est en buis et l'autre en cornouiller ${ }^{12}$.

Le deuxième chnakvi est un peu plus long (environ $51 \mathrm{~cm})^{13}$. Les baguettes sont faites du même bois (probablement du cornouiller); leurs extrémités ne sont pas taillées en pointe, mais semblent avoir été travaillées de manière à être très légèrement arrondies (fig. 4). Elles sont reliées par un lien étroit en tissu vert foncé qui autorise un écart maximal d'environ $5 \mathrm{~cm}$. Sur cet outil, il semble que l'on puisse déterminer des zones d'usure consécutives à l'utilisation, de part et d'autre des baguettes.

21 Ces deux chnakvi sont constitués de baguettes légèrement courbées ou tordues : nous ne savons pas si ces déformations sont originelles ou si elles résultent d'un usage intensif. Ils portent par endroits plusieurs traces manifestes d'usure par polissage. Enfin, ils sont très maniables et leur poids est très faible, de l'ordre de $100 \mathrm{~g}$ seulement (le poids variant plus généralement entre 80 et $90 \mathrm{~g}$ ).

Chaque paysan était capable de fabriquer son propre chnakvi, avec du bois de cornouiller (mais pas exclusivement puisque l'on pouvait employer du buis, entre autres), spécialement choisi pour ses qualités de souplesse et de résistance. Les informations recueillies dans plusieurs des régions du nord-ouest de la Géorgie se recoupent et montrent que les formes comme les dimensions de l'instrument peuvent varier d'une zone géographique -et surtout d'un fabricant- à l'autre. Un chnakvi n'est donc jamais exactement identique à un autre.

Le matériau et la facilité avec lesquels on confectionnait l'outil impliquent sa faible valeur pécuniaire. La cassure de l'un des éléments ou la perte de l'objet n'avait donc pas d'incidence notable et son entretien n'imposait aucune procédure particulière : lorsque l'une des tiges se brisait, elle était remplacée sans tarder. Cela pourrait expliquer pourquoi l'outil conservé au Musée de Tbilissi (cf. supra) est fait de deux baguettes d'un bois différent. Toutes ces raisons semblent rendre compte de la rareté des chnakvi conservés parmi les objets transmis au sein de certaines familles de paysans.

Pourtant, une question se pose quant à la transmission de l'outil d'une saison à l'autre, et deux hypothèses sont possibles: soit l'outil était fabriqué puis jeté après chaque moisson, soit il était fabriqué, utilisé puis conservé jusqu'à la moisson suivante, et on ne le remplaçait que lorsqu'il se cassait. 


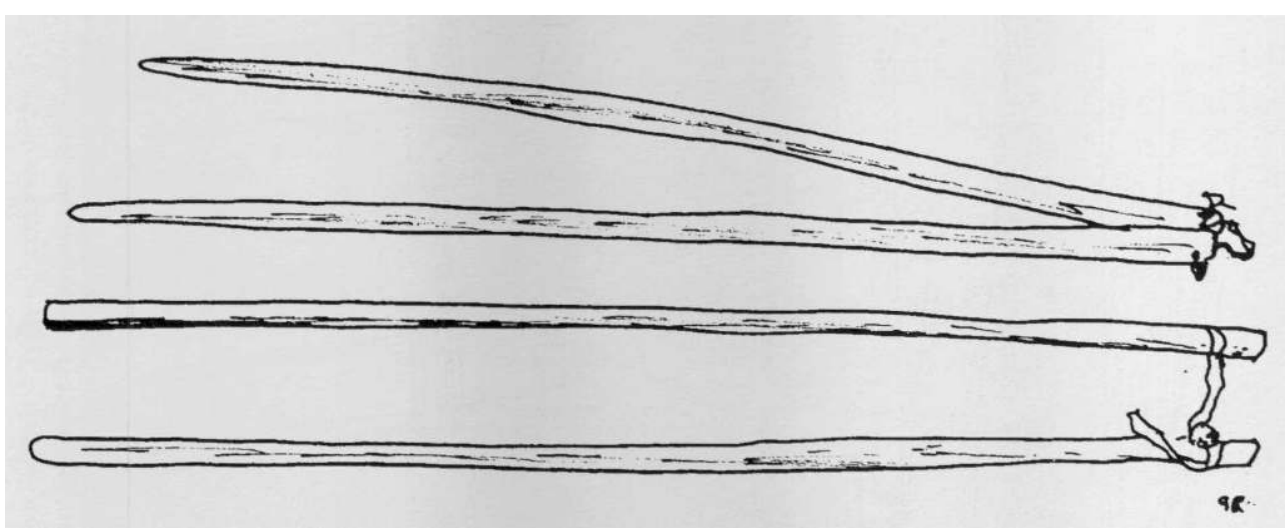

Figure 1. Deux chnakvi - Musée d'Ethnographie d'État Djanachia de Tbilissi (avec autorisation des autorités géorgiennes)
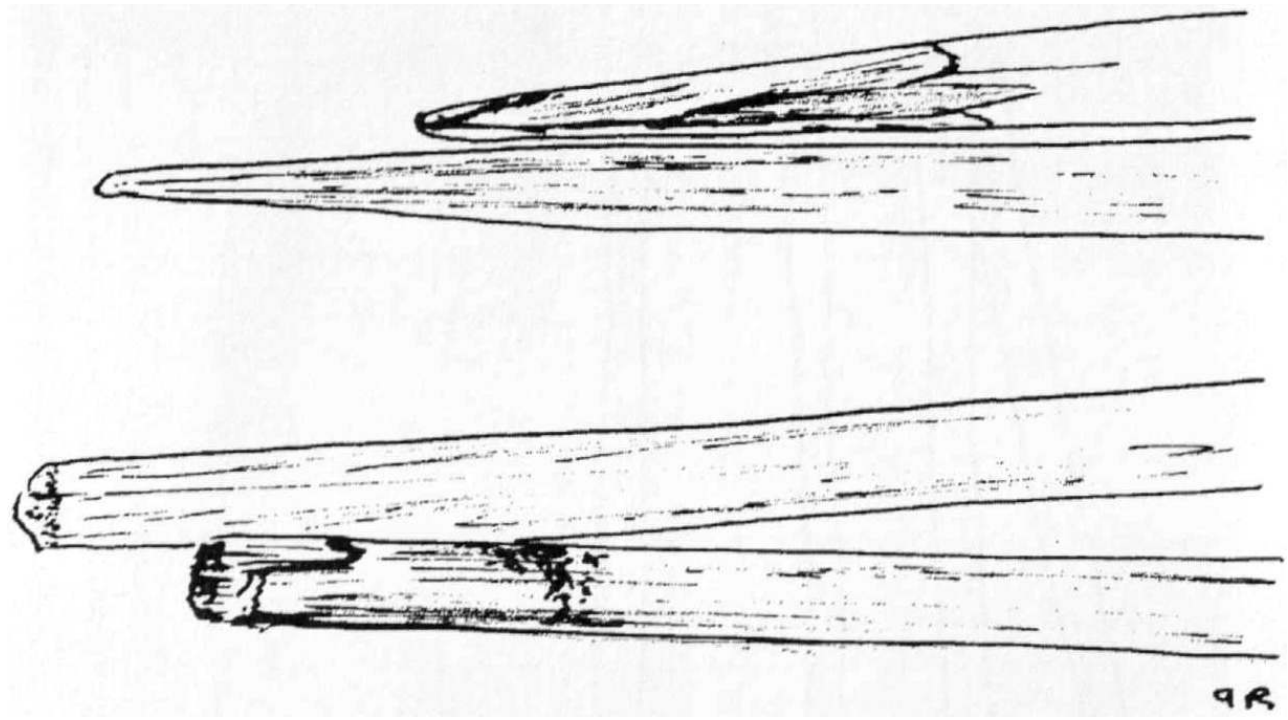

Figure 2. Extrémités mobiles des deux chnakvi - Musée d'Ethnographie de Tbilissi (Géorgie)
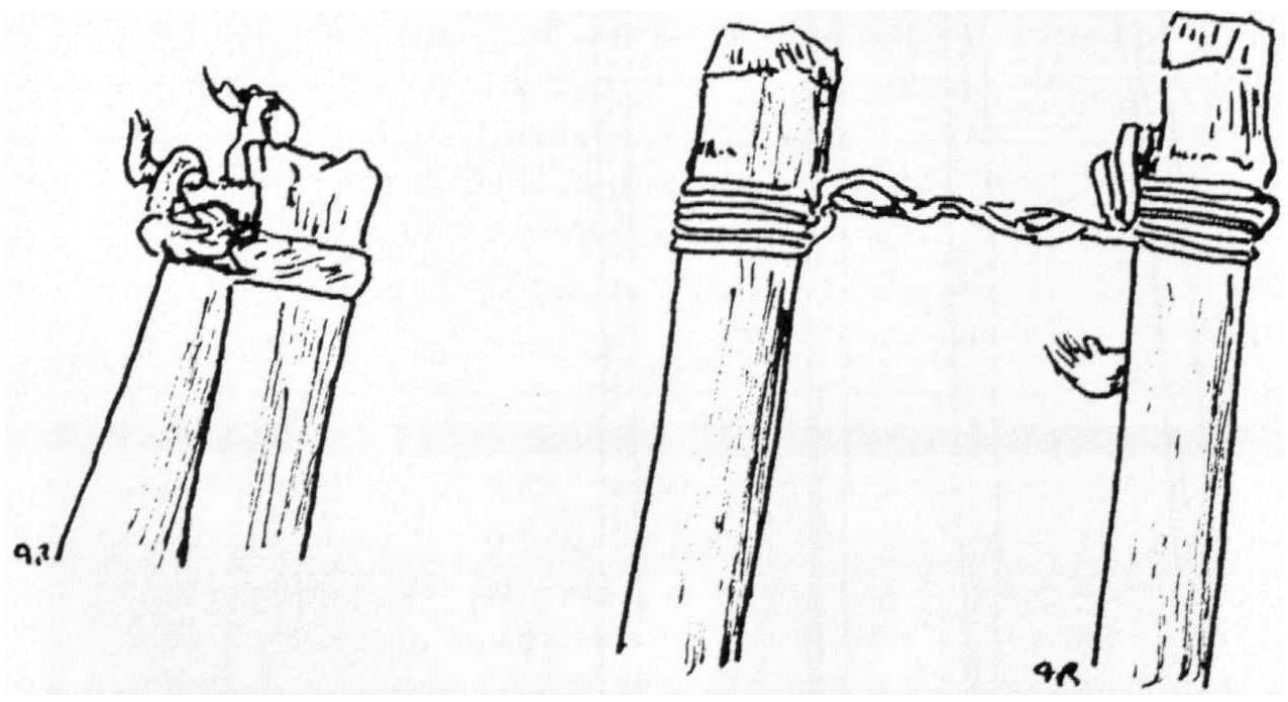

Figure 3. Extrémités attachées des deux chnakvi - Musée d'Ethnographie de Tbilissi (Géorgie) 

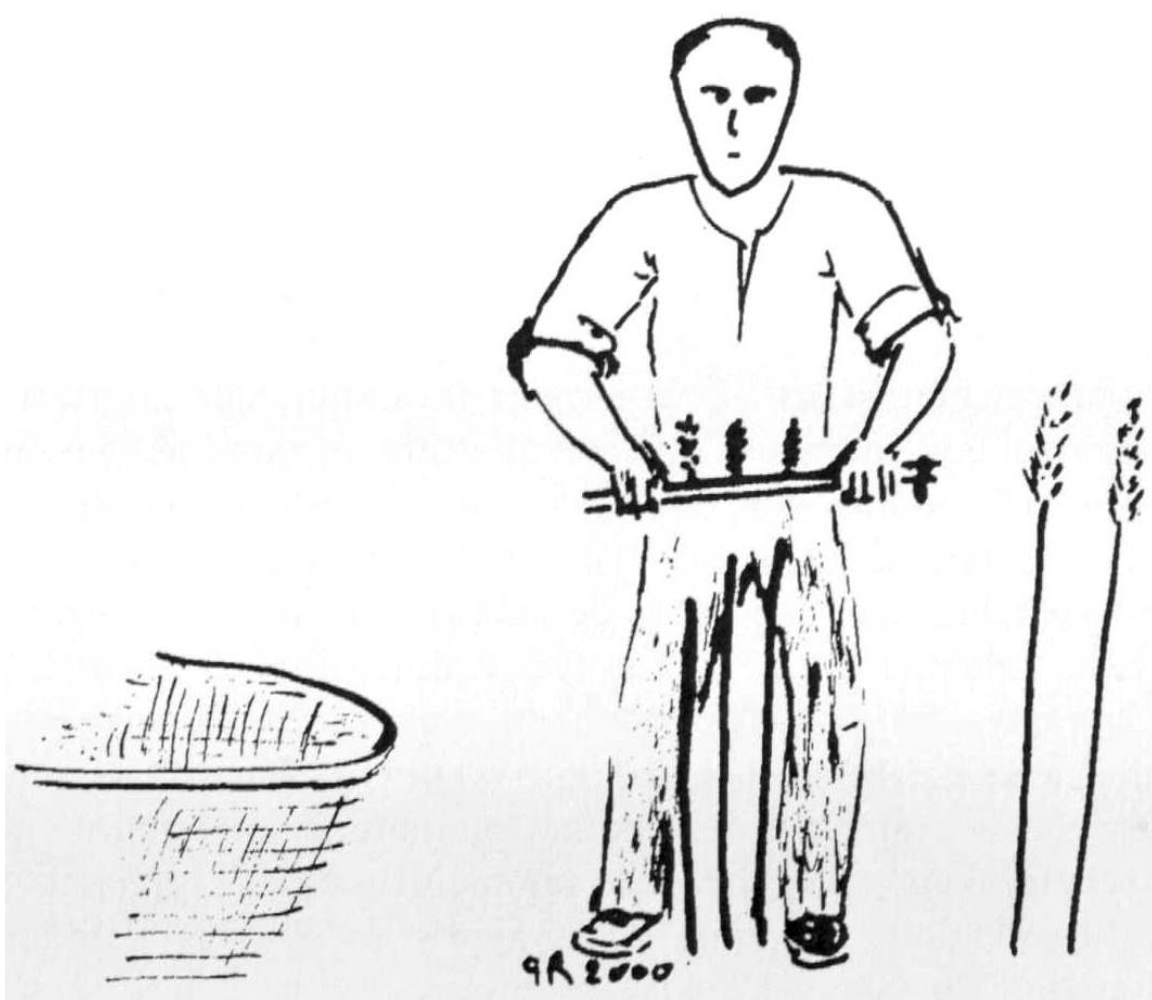

Figure 4. Schéma d'utilisation du chnakvi géorgien

Ainsi, l'un des outils présents au Musée de Tbilissi accuse une importante patine, peutêtre due à des années d'entretien et de conservation muséologique. Mais nous devons nous convaincre que cette patine est davantage le fait d'une transmission familiale : en effet, si l'objet est assurément resté beaucoup plus longtemps dans les réserves de l'établissement qu'il n'a fonctionné dans le contexte de son utilisation même, c'est pourtant pendant cette période que les traces d'usure, de micro-chocs et les rayures ont logiquement été les plus marquantes, puis se sont progressivement imprimées sur le matériau. Les analyses tribologiques comparatives ${ }^{14}$ seront d'ailleurs sans doute en mesure de révéler.

Dans un premier temps, on pourrait penser que rien ne justifiait sa conservation d'une année sur l'autre au sein des familles de paysans. Pourtant, il faut convenir, a contrario, que rien ne prouve que les outils n'étaient pas conservés d'une année sur l'autre. En premier lieu, la patine que nous avons observée a pu en partie trouver son origine dans un polissage effectué au moment de la fabrication; mais nous avons noté certaines marques, traces d'usure ou de probable torsion à l'effort qui semblent plus caractéristiques d'une utilisation dans la durée et qui sont donc sans doute consécutives à un usage répété, à l'exemple des traces de polissage, peut-être dues à de fréquents passages des mains sur les parties préhensiles. Par ailleurs, le fait de conserver ce type d'outil d'une année sur l'autre constitue un choix qui reste bien entendu lié aux qualités mécaniques intrinsèques du bois, qualités qui peuvent ne pas être identiques pour l'objet neuf, fabriqué « en saison ", et pour l'objet ancien, conservé depuis la saison dernière : le bois étant plus sec, l'outil est alors devenu sensiblement moins souple et donc forcément plus résistant. C'est un argument technique qui pourrait justifier que l'on conserve l'outil jusqu'à la moisson suivante, certains témoins nous l'ont d'ailleurs confirmé. Notons que les chnakvi, par leur forme et leur résistance 
précisément, semblent dans ce cas accuser des caractéristiques mécaniques relativement proches des baguettes de moisson en bois utilisées au Népal central ${ }^{15}$ pour désépier l'orge et le blé. Enfin, notre hypothèse pourrait emporter l'agrément en ce qu'un outil, en théorie, est par nature un allié dont l'homme ne se sépare en principe que rarement. Un pas de plus dans le raisonnement justifierait d'opter pour la transmission plutôt que pour l'abandon systématique après chaque saison. Nous devons préciser enfin un élément anthropologique que nous avons appris de certains habitants de la région de Ratcha : en Géorgie, l'outil se respecte, on ne le jette pas, on ne le brûle pas, il se range et on l'abandonne lorsque l'on ne peut pas faire autrement; si possible, on le récupère, en totalité ou en partie, on le réutilise, on tente de prolonger sa durée de vie parce que l'on obéit, là comme ailleurs dans le monde, non seulement à ces exigences pratiques, mais également à certaines croyances.

\section{Manier le chnakvi}

Le chnakvi était utilisé des deux mains, sans gant de protection, le plus souvent par des hommes (mais les femmes pouvaient également s'en servir). On procédait de la manière suivante : dans le champ de céréales, le paysan ouvrait les baguettes entre lesquelles il engageait une certaine quantité de tiges qu'il répartissait ensuite rapidement et presque simultanément sur toute la longueur de l'ouverture. Puis il rapprochait les deux éléments de bois en les ramenant de part et d'autre des végétaux qui étaient ainsi enserrés, de façon très lâche et sans écrasement : les tiges se trouvaient comme guidées dans l'espace étroit formé par les baguettes. Des deux mains, le paysan saisissait alors un peu plus fermement les deux extrémités du chnakvi qui se refermait alors davantage sur les tiges de céréales, toujours sans les presser. Dans un troisième temps, il remontait les baguettes verticalement, perpendiculairement aux tuyaux donc, en effleurant les tiges de bas en haut, afin de rechercher le contact terminal permettant d'arracher (par rupture d'avec leur tige) les épis qui s'étaient alors coincés dans l'étroit espace entre les bois. Deux témoins oculaires âgés nous ont précisé que le paysan pouvait éventuellement -mais cela était très rare- compléter l'action verticale de son geste en pliant légèrement les poignets (d'un geste net) vers l'avant, ce qui contribuait le cas échéant à achever de désolidariser quelques épis récalcitrants des tiges sur lesquelles ils restaient fixés. Le dernier mouvement consistait à déplacer latéralement les bras et à déposer les épis ainsi récoltés dans un panier à large ouverture déposé tout près, en les libérant par désolidarisation des baguettes. Un ethnologue de la région de Ratcha nous a cependant précisé que l'on pouvait également prendre d'une main les épis restés sur les baguettes pour les jeter dans le panier. Il existe de ce fait deux manières de récupérer les épis.

Le geste effectué avec le chnakvi ne demandait nulle force pour assurer le désépiage. Nous avons personnellement manipulé chacune des deux espèces de blé en question, zanduri et makha, dont on nous a d'ailleurs remis un exemplaire ${ }^{16}$, et nous avons été très surpris de constater avec quelle facilité ces épis tombent lorsque la plante est mûre, au point qu'une pression à peine marquée des doigts simplement posés sur le tuyau, à la base de l'épi suffit à assurer la séparation.

29 Les céréales récoltées au moyen des baguettes sont généralement des espèces de blé ou d'orge de montagne, devenues relativement rares. C'est donc à travers les répartitions effectuées par les botanistes que nous pourrons présenter la classification des blés 
généralement admise aujourd'hui. Il existerait à ce jour dans le monde cinq ou six espèces principales, réparties d'après leurs distinctions génétiques, chacune d'elle étant constituée de plusieurs sous-groupes. Cette classification s'opère entre les blés monococcum comprenant 14 chromosomes (blés vêtus et blés engrain), les blés dicoccum, constitués de 28 chromosomes (blés vêtus et blés nus) et les blés tendres disposant de 48 chromosomes et regroupant également des blés vêtus et des blés nus. Plusieurs classifications secondaires évidemment beaucoup plus détaillées sont proposées par les botanistes ou paléobotanistes (Haudricourt \& Hédin 1987; LeroiGourhan 1994 : 144).

\section{L'hypothèse de l'ancienneté}

Dans le cas géorgien, certains historiens pensent que l'usage du chnakvi remonte à l'Antiquité, voire à la préhistoire (néolithique).

31 La première hypothèse retenue découle du fait que l'actuel mot géorgien de « chnakvi » a été étymologiquement rapproché, par les spécialistes, d'un mot issu de la langue Svane, chinakoû, dont nous avons déjà évoqué la signification (cf. supra), cette dernière précision étant implicitement déduite, selon les partisans de cette hypothèse, de la construction idiomatique du mot Svane. Il est vrai que la Svanétie ${ }^{17}$ est l'une des régions les plus anciennes de Géorgie, lovée dans les contreforts élevés des montagnes du Grand Caucase, et qui abrite des populations dont la culture, les coutumes et la langue figurent parmi les plus authentiques de la Géorgie Occidentale du Nord, comme le montre le schéma généalogique des langues kartvéliennes de Deeters -auquel se réfèrent les chercheurs ${ }^{18}$. Mais le terme chinakoû n'établit pas clairement qu'il s'agissait véritablement de tiges de céréales dont on aurait préalablement coupé les seuls épis (on ramassait peut-être des brassées végétales).

La seconde hypothèse dont on se souvient qu'elle rapproche les mots de chanko ou chanki du terme sanki appartenant à la langue akkadienne (cf. supra), laisse également supposer une grande ancienneté des techniques mais pas forcément de l'outil. Ces deux tentatives d'explication renvoient au problème du rapport trans-chronologique qu'il faut trouver entre la description d'un résultat, l'emploi d'une technique et l'usage supposé d'un outil.

33 La plupart des savants géorgiens pensent que les premiers paysans ont arraché les blés avec les tiges et leurs racines, puis qu'ils auraient ensuite enlevé manuellement les épis seuls, les tiges restant sur le champ. Certains auteurs (comme Dzidziguri 1994) suggèrent l'existence des baguettes de récolte dès la période préhistorique, se fondant sur l'hypothèse qu'elles auraient constitué un intermédiaire entre les techniques de récoltes manuelles et celles effectuées avec les outils coupants. Nous devons cependant nuancer cette vision simpliste de la chronologie : sur ce point, nous exprimons une certaine réserve, puisque ce raisonnement impliquerait que les lames ou les éclats retouchés de silex n'étaient pas encore connus lorsque l'homme avait mis au point les baguettes, ce qui est un élément discutable et qui n'est de toute manière fondé sur aucune hypothèse ou point scientifique.

En Caucase, les faucilles n'ont pas fait disparaittre l'arrachage à la main, pas plus que ces deux méthodes n'ont empêché de continuer à employer des baguettes. À l'inverse donc, l'existence des baguettes ne peut logiquement se supposer que dans la durée, sur la base d'un raisonnement hypothético-déductif qui devrait se construire sur une 
déconnexion relative d'avec les techniques connues. De fait, la situation est complexe : les procédés de récolte ont varié en fonction de l'espèce de blé cultivée et l'on ne sait pas depuis quand les outils de récolte en bois sont entrés dans les usages ni combien de pratiques antérieures (et lesquelles), ont existé avant leur mise au point. Par conséquent, il est malaisé de conclure à l'emploi de baguettes à récolter dans la période pré-antique. Toutefois, si l'intuition de l'ancienneté de ce type d'outil reste valable, il semble qu'elle ne doive pas être recherchée systématiquement dans des sources archéologiques pouvant constituer de facto des preuves matérielles, mais impossibles à trouver. Elle doit reposer davantage sur une suite de raisonnements qui, bien que du domaine de la spéculation, s'appuient sur des éléments scientifiquement établis et revêtent, à défaut de certitude, un caractère de plausibilité.

Le fait de trouver des dessins de baguettes de récolte parmi ceux d'autres outils lithiques ou osseux de la culture matérielle néolithique géorgienne dans des travaux récents (Dzidziguri 1994) montre que l'idée est généralement admise aujourd'hui. Notons que les baguettes sont alors, dans le cadre de ces travaux, les seuls outils de bois figurant parmi les dessins d'outils en pierre -alors qu'on n'en a jamais retrouvés en fouilles archéologiques- du contexte chronologique dans lequel cet auteur les place, cela impliquant pour nous, Occidentaux, et par la force des choses, des distinctions fondamentales d'avec les modes opératoires déjà classifiés par André Leroi-Gourhan pour les outils lithiques préhistoriques.

Pour rester objective, notre analyse se fonde sur deux points, l'un théorique, l'autre technique. Sur le plan proprement théorique, il faut tout d'abord souligner que les baguettes à récolter de Géorgie constituent un outil double, élément concret qui contraste au regard de la très forte majorité des outils connus dans la période préhistorique dont elles seraient, pour certains chercheurs, supposées provenir. En ce sens, leur manipulation exige donc une série d'actions ordonnées ${ }^{19}$ (prise des tiges, renfermement, arrachage, rupture et dépose), qui ne sont efficaces et cohérentes que par leur succession puisque l'une ne peut s'effectuer qu'après l'achèvement de la précédente, actions sensiblement différentes de celles, souvent beaucoup plus primaires, constituées par des gestes simples réalisés avec les outils préhistoriques (en pierre, en os...), mieux connus, et dont la triade d'actions pourrait se résumer par les termes : pointe/choc/coupe ${ }^{20}$. À ce titre, les baguettes de récolte sembleraient devoir s'éloigner des périodes très anciennes parce qu'elles impliquent une diversification technique du geste, contrairement à tous les autres outils datant de la préhistoire, un peu comme si elles se plaçaient, par ces seuls critères, hors du cadre technique de cette période (Leroi-Gourhan 1971 : 47-64).

Mais il est vrai que l'on ne peut pas assimiler la classification d'André Leroi-Gourhan à une description très exhaustive de tous les outils préhistoriques, car elle apparaitrait réductrice, alors qu'elle doit au contraire vivre d'un enrichissement par compléments et diversifications. Cette remarque, qui tendrait à désengager la description de l'action des baguettes d'un contexte préhistorique, ne doit pas être retenue puisque certains travaux portant par exemple sur les harpons magdaléniens ont précisément pu montrer, par comparaison technique reposant sur l'ethnologie, qu'il s'agissait d'armes constituées de plusieurs parties et qui exigeaient des gestes différents de ceux énumérés dans la classification d'André Leroi-Gourhan ${ }^{21}$. On retrouve ce type de questions avec le propulseur, qui implique un jet différent à celui du lancer réalisé à la 
main, par exemple. L'outil double ou relativement complexe ${ }^{22}$ existe depuis très longtemps. C'est un premier point.

Sur le plan technique maintenant, les éléments qui plaident en faveur de la thèse de l'ancienneté du chnakvi géorgien sont peu nombreux mais tout à fait plausibles.

En premier lieu, les analyses relatives aux périodes préhistoriques doivent être nuancées, et ce sont dans ces multiples hypothèses que réside la complexité du sujet. Les outils de bois de type bâton à fouir ou bâtons de récolte, les baguettes à récolter le riz sauvage chez les indiens Ojibwas ou celles qui servaient à érusser le riz en Asie du Sud-Est, sont peut-être parmi les plus vieux outils du monde (Reigniez s/presse). Les baguettes à récolter l'orge ou le blé au Népal ou en Asturies supportent la comparaison dans l'histoire des techniques, autant que celles d'un usage équivalent en Géorgie, ces trois dernières régions du monde étant des zones de moyenne ou de haute montagne. Les recherches sont d'autant plus complexes que l'origine même des baguettes de Géorgie reste difficile à cerner puisque l'outil a tout aussi bien pu être créé en Caucase qu'importé d'une autre zone, voire fabriqué sur le modèle d'un objet antérieur, employé dans une autre contrée. Ce sont des hypothèses diffusionnistes qui restent plausibles -et pour l'instant toujours en suspens.

Or, nous savons que la zone sud de la Géorgie a été récemment le théâtre de la découverte de restes d'hommes fossiles datés de - 1,8 million d'années, vestiges mis au jour entre l'été 1999 et l'été 2001 dans la zone de Dmanissi. Selon les hypothèses en vigueur, un groupe proche de l'Homo Ergaster vivant en Afrique de l'Est semble avoir remonté depuis cette région et suivi les chemins de la Syrie, du Liban, de la Turquie, avant de passer au pied des montagnes du petit Caucase, et peut-être de s'y fixer. Le phénomène est essentiel car il prouve que le plus ancien Européen est passé dans le territoire de la Géorgie. Or, entre la datation de ces vestiges humains -les plus anciens d'Europe - et le moment où les premiers agriculteurs sont attestés en Géorgie, il s'écoule plus d'un million sept cent mille ans, ce qui laisse un temps considérable aux premières populations, d'une part pour se fixer, et d'autre part pour concevoir des outils très rudimentaires, lithiques ou monoxyles, puis ceux, beaucoup plus tard, nécessaires à la cueillette des premiers végétaux sauvages récoltés, puis sujets à une proto-agriculture. La base théorique d'une construction par hypothèse de l'ancienneté des baguettes à récolter est désormais fondée par la mise en place d'une conjonction du double facteur [milieu-prédateur humain], ces outils ayant pu apparaître à partir du moment où se vérifie la triple conjonction [milieu-prédateur humain-végétal consommé].

Précisément parce que cette triple conjonction peut remonter à des périodes très lointaines, nous pensons que les baguettes sont fort anciennes et nous partageons en cela les analyses des spécialistes géorgiens, notamment celles de G. Chitaïa, de I. Djavakhichvili, de N. Bregadze et plus récemment de L. Dzidziguri. Nous pouvons ainsi supposer, selon les éléments que les ethnobotanistes ont indiqués, l'outil étant spécifiquement employé pour désépier le zanduri et le makha ${ }^{23}$, que le chnakvi aurait pu théoriquement apparaître pendant le néolithique moyen ou final, périodes où se sont progressivement fixés les premiers habitats qui ont permis une sédentarisation favorisant à son tour l'organisation des travaux de récolte, proto-agricoles et/ou d'élevage, ainsi qu'une très probable réflexion sur les moyens de les mettre en œuvre. Or, si l'on se réfère aux travaux des ethnobotanistes, ces premiers blés zanduri et makha sont attestés dans les premiers temps de l'agriculture en zone de montagne, 
lorsque les peuples sédentarisés ont cherché à cultiver des espèces de céréales dont les qualités (rusticité) pouvaient être exploitées... C'est-à-dire dans la période du néolithique final environ, pour le Caucase. En Svanétie par exemple, le seigle et le millet ont été découverts par D. Menabde en 1967 dans la vallée de Tchouxeti : dans le village de Nakra, les grains voisinaient avec des haches de Colchide que la typologie permet de dater du Bronze tardif, alors que dans le village d'Etseri, les grains ont pu être datés des $1^{\text {er }}$ et $2^{2 \text { ème }}$ millénaires av. J.-C (Tchartonali $\left.1996: 153\right)$.

Autrement dit, conformément à ce schéma, la fixation de l'habitat, aurait propablement précédé la pratique de l'agriculture. Si les baguettes à récolter semblent constituer l'un des premiers outils de bois de la protosédentarisation agricole ${ }^{24}$, rien ne permet pourtant d'affiner notre localisation, et l'hypothèse peut l'inscrire tout aussi bien dans des périodes sensiblement plus tardives mais voisines, l'Enéolithique, voire le Bronze. Le raisonnement dans la durée ne devant pas contraindre à définir le cadre de trop strictes frontières chronologiques ${ }^{25}$.

Nous savons que certains outils, comme des couteaux en silex, existaient en Géorgie depuis les $V^{\text {ème }}$ et $I^{\text {ème }}$ millénaires, ainsi que des faucilles fabriquées au moyen de mâchoires inférieures d'animaux édentées, dont les cavités permettaient d'enchâsser et de fixer, avec de la résine naturelle (ou du goudron), des lames de silex retouchées. Le Bronze et le Fer ont également donné naissance à des outils agricoles et nous pouvons supposer que certains d'entre eux ont pu servir à la récolte de végétaux et de céréales dans les débuts de l'agriculture caucasienne ${ }^{26}$. Nous pouvons donc avancer l'hypothèse selon laquelle les baguettes ont été mises au point indépendamment, pour assurer une opération que ces outils lithiques, dont l'action mécanique était tout à fait différente, ne permettaient peut-être pas de réaliser. Les recherches archéologiques menées en Caucase prouvent par ailleurs que, dans les périodes pré-antiques, on employait toujours des outils de pierre, d'os ou de bois dans l'agriculture, quelques types d'instruments à tête de métal ayant fait ensuite leur apparition ${ }^{27}$. Le fait que les baguettes étaient toujours en usage au $\mathrm{XX}^{\mathrm{e}}$ siècle, quand les outils de métal existaient depuis longtemps, montre à l'évidence que ces derniers n'étaient pas estimés performants pour réaliser un certain type de travail.

Ainsi, la transmission du chnakvi pourrait être supposée depuis ces périodes préantiques, dans les régions de culture des plantes particulières qu'il servait à récolter, jusque dans la période contemporaine, sans évoluer ni dans sa forme, ni dans sa fonction.

En ce sens justement, nous relèverons le fait que la persistance d'un outil aussi simple n'implique pas de transformation majeure ni dans la forme ni dans les dimensions de l'objet, ce facteur pouvant alors accréditer la thèse de la pérennité de la forme basique dans le temps, et lui associer par répercussion l'implication probable d'une absence de nécessité d'en améliorer les gestes, sans doute déjà parfaitement maîtrisés.

Et cela est finalement assez logique puisque l'évolution de la forme de l'outil a une incidence sur le geste et qu'inversement, une tentative d'amélioration du geste peut avoir certaines conséquences sur la forme de l'outil. Nous pouvons donc légitimement supposer que la recherche d'évolution alternative ${ }^{28}$ ou bilatérale ${ }^{29} \mathrm{du}$ chnakvi géorgien ${ }^{30} \mathrm{a}$ été abandonnée très tôt dans le temps, parce que probablement estimée comme n'étant déjà plus nécessaire à poursuivre.

Or, c'est parce que le chnakvi offre une performance mécanique reconnue comme irremplaçable et inégalable, parfaitement adaptée, qu'il n'a jamais été remplacé par un 
autre outil : c'est ce constat qui nous renvoie à l'hypothèse de la probable ancienneté de l'objet, et de sa possible mise au point précisément justifiée par la nécessité de son utilisation dans la récolte des céréales qu'il servait à travailler (affirmation du lien outil-plante). Il faudrait probablement formuler la même remarque quant au Népal et aux Asturies.

Nous supposons par conséquent l'ancienneté probable des baguettes à désépier de Géorgie par le phénomène de la simultanéité des techniques, qui implique que la mise au point d'une technique, d'une opération ou d'un outil ne fait pas obligatoirement disparaître sur l'instant les anciennes techniques, opérations ou outils usités jusque-là. Une utilisation simultanée des techniques, qui se superposent donc dans le cadre de leur propre évolution, sans se détruire forcément, reste l'hypothèse qui semble la plus vraisemblable : dans ce schéma, le chnakvi géorgien tient parfaitement sa place.

Les baguettes à moissonner le blé ou l'orge sont attestées seulement en trois endroits du monde : a u Népal et dans le nord de l'Espagne, ainsi que dans le Caucase. Nous avons recherché en Géorgie des éléments sur cet outil double, à la fabrication très simple mais à la manipulation visiblement marginale par rapport aux autres outils de récolte conventionnels. Cette enquête nous a confronté à une réalité que bien d'autres pays connaissent : avec la modernité, nombre d'outils disparaissent et, avec eux, tout un savoir-faire dont on ne retrouve des traces que dans certains écrits ou grâce aux témoignages. C'est le cas du chnakvi.

51 Avec un objet de ce type, nous constatons d'abord qu'il n'y a pas de correspondance systématique entre la simplicité d'un outil et les actions qu'il implique. Les gestes servant à actionner les baguettes à moissonner sont particuliers, et l'outil lui-même l'est au point de ne pouvoir être précisément localisé dans la chronologie des techniques de récolte. On se souviendra par ailleurs que cette technique se rapproche sensiblement d'une autre, pratiquée dans la même région du Caucase mais pour une autre activité, la récolte du miel : il y a donc là un rapport réel, celui-ci pouvant peutêtre fournir sur l'une ou l'autre technique davantage d'informations.

Enfin, les exemples attestés en dehors du domaine caucasien montrent que les baguettes à moissonner par désépiage constituent un témoignage anthropologique très concret de l'aptitude d'une population à résoudre, lors d'une période donnée, un problème particulier posé par un végétal fragile et difficile à travailler. Il s'agit ici d'un cas identifié où l'outil de bois a été précisément créé en complément d'autres outils agricoles (en pierre, en os ou en métal selon l'époque de son apparition). En Géorgie, comme au Népal ou dans le nord de l'Espagne, l'usage de ces baguettes s'est prolongé jusque dans le milieu du $\mathrm{XX}^{\mathrm{e}}$ siècle, malgré l'apparition de techniques différentes. Cela constitue peut-être l'un des très rares exemples qui montre qu'un outil agricole individuel de facture archaïsante et de conception élémentaire est resté, dans des conditions à dire vrai bien précises, irremplaçable et d'ailleurs jamais remplacé, ni dans son matériau, ni dans sa fonction. 


\section{BIBLIOGRAPHIE}

Bregadze, Nelli

1982a, Essai sur l'agro-ethnographie de la Géorgie. Tbilissi : Éditions Menierba (en russe).

1982b, Atlas historico-ethnographique de la Géorgie, les matériaux. Tbilissi : Institut d'Histoire et d'archéologie Ivane Djavachichvili (en géorgien).

Chitaïa, Georges

1980, Atlas historico-ethnographique de la Géorgie, les matériaux. Tbilissi : Études Scientifiques (en Géorgien).

1987, L'outil de Colchide, le chnakvi, Travaux. Tbilissi : Éditions Sciences (en géorgien).

Dekaprelevich (L.A.) et Menabde (V.L.)

1932, Journal of Applied Botany, 5, 1.

Dzidziguri, Lasha

1994, From the history of ancient Colchis agriculture. Tbilissi : Éditions Sciences.

Haudricourt, André-Georges \& Louis Hedin

1987, L’homme et les plantes cultivées. Paris : A. M. Métailié.

Leroi-Gourhan, André

1971, L'homme et la matière. Paris : Albin Michel.

1994, Dictionnaire de la Préhistoire. Paris : Presses Universitaires de France.

Reigniez, Pascal

s/presse, Les outils de bois - approche anthropologique (titre provisoire).

Sigaut, François

1978, «Identification des techniques de récolte des graines alimentaires », Journal d'Agriculture Traditionnelle et de Botanique Appliquée XXV, 3 : 145-161.

Sigaut, François, G. Ortiz \& Gérard Toffin

1980, « Moissons sans faucille, Asturies et Népal », Conférences de la Société d'Ethnozoologie et d'Ethnobotanique, séance du 8 nov 1979, Laboratoire d'Ethnobotanique et d'Ethnozoologie du Muséum d'Histoire Naturelle, Bulletin de liaison $n^{\circ}$ 8, mai 1980, pp. 2-4.

Steensberg, Axel

1943, Ancient harvesting implements, a study in archaeology and human geography. Copenhagen :

Nationalmuseets Sktifter, arkacologisk-histotisk, Raekke.

Tchartolani, Chota

1996, L'ancienne Svaneti. Tbilissi.

Toffin, Gérard

1983, « Moisson aux baguettes au Népal central », Objets et Mondes 33 (3-4) : 173-176.

\section{ANNEXES}

En 1980, l'académicien géorgien G. Chitaïa notait qu'il existe 16 espèces de blé répandues dans le monde, dont 12 attestées en Géorgie, plusieurs d'entre elles (six) étant endémiques, d'autres étant constituées de formes initiales cultivées dans des 
périodes pré-antiques et certaines enfin de blés sauvages propres ou non à cette zone géographique (Chitala 1980 : 74 ; Bregadze 1982b : 46). Cette grande région sud du Caucase aurait été, selon l'ethnobotaniste russe Nicolas Vavilov' ${ }^{31}$, l'un des six foyers primaires de culture du blé dans le monde. Il est vrai que les débuts de l'agriculture dans le domaine paléo-caucasien incluant le sud du Caucase, l'Arménie et l'Anatolie, a constitué pendant des millénaires une zone de croisement et d'échanges dont on peut penser que les répercussions culturelles ont pu également concerner en partie les plantes cultivées. La question se pose ouvertement avec certaines variétés dont on ne parvient plus précisément à déterminer si elles ont été créées par croisement délibéré avec d'autres, ou bien issues de mutations génétiques d'origine naturelle. Parmi les blés identifiés, deux doivent donc plus particulièrement retenir ici notre attention parce qu'ils ont été, jusqu'à une période récente, principalement récoltés en Géorgie au moyen du chnakvi : il s'agit des espèces zanduri et makha.

Le zanduri (Triticum timopheevii) serait un blé hybride provenant du croisement d'un blé engrain et d'une espèce endémique au point qu'il est difficile de déterminer s'il est réellement domestique ou sauvage (Haudricourt \& Hedin 1987 : 134). Le blé makha pose en ce sens le même problème, puisque l'on ne saurait dire aujourd'hui avec précision s'il est issu du croisement d'un blé tendre avec l'amidonnier ou bien d'une mutation (voulue ou non) de l'une des nombreuses espèces de blé tendre cultivées dans le Caucase (idem). Il est en tout cas très intétessant de noter que les spécialistes ont observé que ces deux végétaux sont toujours présents dans les hautes régions de Ratcha et de Lechkhumi, c'est-à-dire plus précisément dans la partie nord-nord/ouest de la Géorgie, même s'ils ne sont plus cultivés autant qu'ils l'étaient autrefois. Il faut noter que ces deux espèces étaient assez endurantes et peu exigeantes, au point que les sols relativement pauvres des régions montagneuses leur convenaient parfaitement.

Ces blés portent encore l'une des caractéristiques des espèces non sauvages de culture primaire, c'est-à-dire que leurs tiges sont à la fois résistantes sur presque toute leur hauteur et cependant très cassantes juste sous les épis. Le moment venu, lorsque les plantes sont mûres, les épis tombent d'eux-mêmes et partant, les grains se ressèment seuls, ce qui assure une régénération autonome de la plante. Il s'agit là d'un paléovestige considéré comme important selon les Caucasiens, qui dénomment d'ailleurs localement ces deux céréales, précisément pour cette raison, blés « cassetête ». Elles ont été cultivées de façon importante jusque dans la première moitié du $\mathrm{XX}^{\mathrm{e}}$ siècle, en grande partie pour leur qualité aromatique puisque l'on en faisait par exemple des pains assez appréciés, dans les régions haute et basse de Ratcha, au nordouest du pays. 11 conviendra d'ajouter pour être très précis que ces pains, qui se conservaient très bien, étaient souvent emportés par ceux qui partaient en campagne de chasse (ou de guerre), ou pour un voyage de plusieurs jours. 


\begin{tabular}{|c|c|c|c|c|c|c|c|c|c|}
\hline $\begin{array}{l}\text { Nom de } \\
\text { l'outil }\end{array}$ & Pays & $\begin{array}{l}\text { Végétal ou } \\
\text { produrit } \\
\text { travaillé }\end{array}$ & $\begin{array}{l}\text { Nombre de } \\
\text { baguetles }\end{array}$ & $\begin{array}{c}\text { Longueur des } \\
\text { baguettes }\end{array}$ & $\begin{array}{c}\text { Section } \\
\text { des } \\
\text { baguetes }\end{array}$ & Matériau & $\begin{array}{l}\text { Poids } \\
\text { (env.) }\end{array}$ & $\begin{array}{l}\text { Nature du lien } \\
\text { et écartement } \\
\text { maximal au } \\
\text { lien }\end{array}$ & $\begin{array}{l}\text { Présence } \\
\text { d'un } \\
\text { décor }\end{array}$ \\
\hline Chnakvi & $\begin{array}{l}\text { (iéoryie, région } \\
\text { de Ratcha zone } \\
\text { de montagne }\end{array}$ & $\begin{array}{c}\text { blé } \\
\text { (zanduri et } \\
\text { makha) }\end{array}$ & 2 & $\begin{array}{l}0.50 \mathrm{~m} \\
\text { environ }\end{array}$ & $\begin{array}{l}\text { dc } 1 \mathrm{à} \\
1,5 \mathrm{~cm}\end{array}$ & $\begin{array}{l}\text { Bois (buis ou } \\
\text { comouiller) }\end{array}$ & $\begin{array}{l}\text { de } 80 \mathrm{à} \\
100 \mathrm{gr} .\end{array}$ & $\begin{array}{l}\text { tissu ou } \\
\text { cordelette } \\
\text {-de } 5 \mathrm{~cm} \mathrm{à} \\
8 \mathrm{~cm}\end{array}$ & non \\
\hline artochani & $\begin{array}{c}\text { Géorgie. région } \\
\text { dAdjarie zone de } \\
\text { montagne }\end{array}$ & $\begin{array}{l}\text { blé "kaci } \\
\text { pouri" }\end{array}$ & 2 & \begin{tabular}{|l|} 
entre $0.25 \mathrm{~m}$ \\
et $0.35 \mathrm{~m}$
\end{tabular} & $\begin{array}{l}\text { de } 1 \text { à } \\
1,5 \mathrm{~cm}\end{array}$ & $\begin{array}{c}\text { bois (épicća, } \\
\text { châtaignier hêtré) }\end{array}$ & $\begin{array}{l}\mathrm{dc} 60 \mathrm{gr} \\
\text { à } 70 \mathrm{gr}\end{array}$ & & non \\
\hline $\begin{array}{c}\text { chanko } \\
\text { ou } \\
\text { chanki }\end{array}$ & $\begin{array}{l}\text { Géorgie, région } \\
\text { de Letchkoumi } \\
\text { Gourie. } \\
\text { Mingrélie, zones } \\
\text { de taible altitude }\end{array}$ & miel & 2 & $\begin{array}{l}\text { entre } 0.35 \mathrm{~m} \\
\text { et } 0.40 \mathrm{~m}\end{array}$ & $\begin{array}{l}\text { de } 1 \text { à } \\
1,5 \mathrm{~cm}\end{array}$ & bois & $\begin{array}{l}\text { de } 50 \mathrm{gr} \\
\text { à } 60 \mathrm{gr}\end{array}$ & Cordelette & non \\
\hline $\begin{array}{c}\text { chanko } \\
\text { ou } \\
\text { chanki }\end{array}$ & $\begin{array}{c}\text { Géorgic. région de } \\
\text { Letchkoumi } \\
\text { Gourie. } \\
\text { Mingrélie, zones } \\
\text { de faible altitude }\end{array}$ & cire & 3 & $\begin{array}{c}\text { entre } 0,35 \mathrm{~m} \\
\text { et } 0,40 \mathrm{~m}\end{array}$ & $\begin{array}{l}\mathrm{dc} 1 \mathrm{à} \\
1,5 \mathrm{~cm}\end{array}$ & bois & $\begin{array}{l}\text { de } 100 \mathrm{gr} \\
\text { à } 120 \mathrm{gr}\end{array}$ & $\begin{array}{c}\text { Cordelette } \\
\text { un peu serrée }\end{array}$ & non \\
\hline
\end{tabular}

\section{NOTES}

2. Membre de la Communauté des Étals Indépendants de l'ex-URSS.

3. Le verbe « érusser » est un mot dialectal de l'ouest de la France provenant de l'ancien français « ruisse » (ronce, broussaille), qui signifie : «...pincer la tige d'un végétal pour en arracher les feuilles ou les graines... » (Grand Dictionnaire Larousse, édition 1983, page 3870).

4. Terme que nous avons créé en le construisant comme le mot désherbage (verbe désherber).

5. Nous remercions Monsieur Guia Berichvili, chercheur titulaire au Musée d'Ethnographie et d'Histoire de la ville d'Oni, région de Ratcha Haute, Géorgie, pour l'entretien qu'il a aimablement accepté de nous accorder en juin 1997. En nous décrivant cette technique, il pensait à un tissu dont la maille était solide et très aérée.

6. Comme leur répartition semble le suggérer (voir carte), il est fort probable que les localisations des techniques présentées soient des foyers résiduels d'un usage plus général en Géorgie: vestiges d'activités en œuvre il y a plusieurs siècles, et même, peut-être, dans la grande région du Caucase.

7. Très modestement et à la suite des savants qui nous ont précédé.

8. Il a travaillé à l'Institut de Botanique Appliquée de Léningrad (aujourd'hui Saint-Petersbourg).

9. Langue sémitique ancienne parlée en Assyrie; Sargon l'Ancien fut le fondateur du royaume d'Akkad, vers le XXIII' s av. J.-C.

10. Il convient par exemple ici de faire la distinction entre couper et détacher par arrachement, de savoir ce que ces termes signifiaient pour les populations d'alors, et de tenir compte de l'évolution du signifiant et du signifié au fil du temps ou même des changements consécutifs aux emprunts des termes d'une langue dans une autre.

11. Nous adressons ici nos remerciements à l'ensemble des Professeurs et Chercheurs du Département d'Ethnographie du Musée d'Ethnographie de Tbilissi, à M. Koté Cholokachvili, ainsi qu'à M. Gouram Gassitachvili, ethnologue, et son équipe, pour leur coopération scientifique.

12. Ce qui dénote, comme nous le verrons un peu plus loin, une probable réparation de l'outil.

13. Il n'est pas possible de déterminer précisément si le chnakvi taillé dont nous parlons était, à l'origine d'une longueur équivalente à l'autre, ou bien si les deux outils avaient dès leur fabrication des longueurs différentes. Les témoignages ont cependant confirmé que les longueurs variaient sensiblement d'un chnakvi à l'autre.

14. Réalisées sur des outils reproduits à l'identique sur le modèle original.

15. Il existait des baguettes en bois et en bambou (cf. infra). 
16. Nous remercions Monsieur Guia Berichvili, de Oni (cf. supra), pour nous avoir donné un exemplaire d'épi des variétés identifiées par lui comme étant des blés makha, khoulougo, zanduri et ipkhli.

17. Ou Souanétie dans les anciens textes.

18. Georgien, Schätze aus dem land des goldenen vlies, Katalog der Ausstellung des Deutschen Bergbau-Museums Bochum in Verbindung mit dem Zentrum für Archäologische Forschungen der Georgischen Akademie der Wissenschaften Tbilissi vom 28/10/2001 bis 19/05/2002. (Bochum $2001: 7)$.

19. Que nous appelons des chaînes d'actions.

20. Avec certaines exceptions cependant, notamment en ce qui concerne la taille des objets lithiques, dont on sait qu'elle nécessitait une différenciation dans le choix du matériau du percuteur, du matériau taillé, éventuellement de sa chauffe, puis dans son positionnement en frappe, puis dans la modulation de la puissance même de cette action.

21. Le harpon étant parfois constitué d'un embout mobile fixé sur une hampe, qui s'en détache lorsqu'il se plante dans la proie. Cela implique une diversité et une multiplicité dans la conception des mécanismes de son action. Nous ne sommes plus dans le cadre des actions simples mais dans celui des chaînes d'action, et de plus, il nous faut prendre en compte dans cet exemple le fait que l'arme elle-même agit indépendamment à un moment donné (entrée dans les chairs, détachement de la pointe du harpon, accrochage dans les chairs et traction). Cf travaux de Michèle Julien (dates ? ?).

22. Par rapport à l'outil d'emploi plus simple, plus primaire. Il convient par ailleurs de distinguer la fabrication de l'outil (qui peut être simple ou complexe) de son utilisation (qui peut également s'avérer simple ou complexe).

23. En n'oubliant pas l'artochani servant à désépier le khaci-pouri.

24. Comme certains types de bâton à fouir, destinés ailleurs à la proto-agriculture.

25. C'est le temps long du présent ethnographique sur lequel raisonnent les ethnologues.

26. Nous ne pensons pas que toutes les faucilles pré-antiques (en os, en silex ou en métal) aient servi à couper des céréales : certaines d'entre elles ont pu servir à couper seulement des herbes, des petites branches arbustives et, le cas échéant en effet, des céréales. Mais l'association «faucille pré-antique » - « céréale » ne doit pas être systématique.

27. Soulignons sur ce point que si un jour l'existence du chnakvi est prouvée dans la période néolithique, elle laisserait entrevoir très tôt une complexité dans l'histoire des techniques de l'agriculture parce que, inévitablement, nous serions amenés à observer non pas une unité de raisonnement face au problème de la récolte, mais une diversité de tentatives qui auraient alors répondu aux particularités propres à telle ou telle espèce de blé, les unes pouvant être sciées à la faucille, les autres arrachées avec des baguettes, et cela dans le cadre de l'activité technique du groupe humain concerné. Ce domaine de diversification technique reste évidemment subordonné aux découvertes de l'archéologie, sans le secours desquelles il ne trouvera jamais d'écho véritablement crédible. Cependant là encore, l'hypothèse est rendue plausible par l'expérience en matière agricole et à l'ancienneté de ces pratiques dans cette région bien définie du Caucase

28. C'est-à-dire commençant soit par l'amélioration du geste, soit par celle de l'outil, chacune ayant ensuite une incidence sur l'autre composante.

29. C'est-à-dire commençant par la recherche conjointe de l'amélioration de l'outil et du geste, les deux composantes étant alors susceptibles de changer en même temps selon les corrections critiques de l'utilisateur.

30. Comme les autres baguettes à désépier du Népal ou des Asturies ou encore celles servant à récolter et constituant d'autres familles typologiques utilisées dans le monde.

31. Grand spécialiste des céréales de l'Institut de Botanique Appliquée de Léningrad (depuis 1991 Saint Pétersbourg). 


\section{RÉSUMÉS}

Il a existé en Géorgie un outil de récolte pour le blé, le chnakvi, que l'on pourrait traduire par «baguettes à moissonner ". L'outil est utilisé dans certaines régions seulement pour les espèces de blé makha et zanduri, peut-être aussi pour un blé nommé khaci pouri et pour la récolte du miel. Ce type d'objet est rare. Par suite, il convient alors de le décrire avec précision, puis de chercher à travers les sources qui le mentionnent, comment il doit être situé dans la chronologie des techniques.

There existed in Georgia a wheat harvesting tool called the chnakvi, better known in western Europe as the "reaping stick». This tool is used in some parts of this country only for special sorts of wheat known as makha and zanduri, and perhaps also for a wheat called khaci pouri and for honey collection. As this sort of tool is tare, it is advisable to describe it with precision, then to look for through the sources which mention it to establish how it must be placed in the chronology of technnology.

Existía en Georgia un instrumento que su usaba para la cosecha del trigo. Se trata del chnakvi, lo que se podría traducir por «varilla de cosechar ». El chnakvi se empleaba (solo en algunas zonas) para recolectar especies genuinas de trigo: makha zanduri, y posiblemente para el trigo khaci pouri, además de la recolección de la miel. Este tipo de objeto es poco frecuente. Conviene pues describirlo con precisión e investigar las fuentes que lo atañen, para situarlo en la cronología de las técnicas.

\section{INDEX}

Mots-clés : blé, Caucase, Géorgie, orge, outil

\section{AUTEUR}

\section{PASCAL REIGNIEZ}

Chercheur associé CNRS, Centre d'Ethnologie Française, Musée National des Arts et Traditions

Populaires 6, Avenue du Mahatma Gandhi 75016 Paris 